\title{
Epidemiology of Influenza in Pregnant Women of the State of Mexico: Season Analysis 2009-2010 To 2018-2019
}

\author{
José de Jesús Coria Lorenzo ${ }^{1 *}$, Enrique Rafael Ortiz García ${ }^{2}$, Jesús Armando Coria Guerrero ${ }^{3}$, Xochitl Mirón \\ Calderón ${ }^{4}$ and Evelyn Pla Esquivel ${ }^{5}$
}

${ }^{1}$ Department of Infectology, Children's Hospital, Federico Gómez, Mexico

${ }^{2}$ Department of Infectology, Instituto Materno Infantil del Estado de Mexico, Mexico

${ }^{3}$ Department of Gynecology, Hospital Municipal "Mariano Matamoros" Bicentenario, ISEM, Mexico

${ }^{4}$ Department of Communicable Diseases and Mycobacteriosis, Epidemiology Department of the ISEM. (Health Institute of the State of Mexico), Mexico.

${ }^{5}$ Department of Influenza Program of Epidemiology, Health Institute of the State of Mexico, Mexico.

Submission: November 11, 2019; Published: November 14, 2019

*Corresponding author: José de Jesús Coria Lorenzo, Department of Infectology, Children’s Hospital, Federico Gómez, Mexico

\begin{abstract}
Objetive: To know the epidemiology of pregnant women in different seasons of influenza in the State of Mexico, from the pandemic to the 2018-2019 season; analyze the group of pregnant women vaccinated vs unvaccinated and who of them got sick of influenza and needed hospitalization, her positivity to influenza virus and co-morbidities.

Material and Methods: A review of cases that were positive for some type of virus was carried out of both the SINAVE-SISVEFLU platform, as well as the concentrates that are in the ISEM epidemiology department.

Results: We found that there were a total of 18971 suspected cases, of which 9826 were women, resulting in only 10 years, $2.8 \%$ of pregnant women (276 pregnancies). The numbers of pregnant women showed a very low prevalence in all seasons, and the global prevalence was of 0.028. Regarding the vaccination of the 276 pregnant women, $186(67.3 \%)$ were not vaccinated vs of the vaccinated women who were only $82(29.7 \%)$, resulting in a percentage of $29.7 \%$ of total (276 pregnant), of which $81 / 82$ (98.7\%) were positive for some type of virus vs not vaccinated against influenza, of which 68 (36.5\% [68/186]) were positive for some type of virus, predominating pandemic influenza AH1N1 in both groups. Regarding the impact of the disease that represented the risk of meriting the hospitalization of all those pregnant women with suspected influenza vaccinated or not, we observed that 49 of the 82 vaccinated were hospitalized 49 (59.7\%) while with the unvaccinated (186) was a higher rate of hospitalization (130 [69.8\%]).
\end{abstract}

Analysis: we observe that although it is true the largest number corresponded to the unvaccinated (130/186 [69.8\%]) vs the vaccinated (49/82 [59.7\%]), it should be noted that practically 49 (100\%) hospitalized were positive for some type of influenza virus unlike those not vaccinated who only $52.3 \%$ were positive for some type of virus, which seems to indicate that the vaccine does not reduce the risk of hospitalization associated with influenza.

Conclusions: Our data obtained suggest that receiving an influenza vaccine does not reduce the risk of getting sick influenza in pregnant women, and nor does it reduce the risk of hospitalization in this group.

Keywords: Influenza; Pandemic; Seasonal; Women; Pregnancy; Hospitalization Estate of México

\section{Introduction}

Influenza virus infection is very common and represents a significant cause of morbidity in specific populations such as pregnant women. In this regard, influenza AH1N1, unlike other types of influenza viruses, is more likely to cause serious illness in pregnant women than in women of reproductive age who are not pregnant. Changes in the immune system, in the heart and in the lungs during pregnancy make pregnant women and even in the puerperium (up to two weeks after childbirth), more likely to get sick with influenza and suffer serious diseases from it, which result in hospitalization for cardio-respiratory complications (mainly associated with bacterial pneumonia, which incidence has been reported from $0.04 \%$ to $1 \%$, and even death), that could endanger their life [1-2]. Physiological changes (which are nor- 
mal [like all changes seen during pregnancy]), such as the fact that ciliary activity is decreased and pulmonary circulatory flow is increased, coupled with the fact that the growing fetus compresses the lungs, by making press up, reducing lung capacity and hence the ability of the lungs to expel fluids; they are combined to favor a greater susceptibility to acquiring influenza and a greater risk of complicating with a bacterial pneumonia picture. Immunological (equally normal) changes during pregnancy such as Absence of blocking antibodies, decrease in the cell-mediated immune reaction, especially NK cells, and also the decrease in the immune response mediated by gestational hormones (chorionic gonadotrophin, lactogen placental, progesterone, and prolactin), determine a lower activity against AH1N1. Therefore, they favor a higher risk of catching influenza during the high season $[3,4]$.

On the other hand it is known that influenza in a pregnant woman can also cause damage to the developing baby. A common symptom of influenza is fever, which has been seen, may be associated with neural tube defects. Other adverse outcomes for a developing baby whose mother acquires influenza are those that increase the chances of premature rupture of membranes, fetal distress, fetal death, spontaneous abortion, premature delivery and low birth weight, also if the mother is with a certain degree of anemia during pregnancy which is aggravated if there is a pneumonic process (it has been reported up to $<10 \mathrm{~g} / \mathrm{dL}$ ), the neonate may also be born anemic [1,5-7].

The first lethal case of respiratory infection by H1N1 in a pregnant woman in the United States was reported in 2009 [8]. In our country during the 2009-2010 pandemic at the Dr. Aurelio Valdivieso General Hospital, in the city of Oaxaca, 13 cases of AH1N1 influenza were detected, corroborated by RT-PCR in specialized laboratories in Canada. During that period 27 suspicious cases were found in pregnant women who went to the Emergency Department, who were tested quickly and a positive result was obtained; however, only ten cases were able to document the infection by viral culture; the rest of the cases were negative and only merited outpatient treatment. In three cases the rapid test was negative, but due to the symptoms, the patients were hospitalized and treated because the following control of cultivation viral was positive. The cumulative incidence was 1.6 per 1000 pregnant women during the period studied. The probability of getting sick with influenza AH1N1 for pregnant women at Dr. Aurelio Valdivieso General Hospital was 1.6 per thousand who entered the institution [9]. In another similar study conducted in the State of Mexico by Coria et al, a review of the three-year annual concentrates (2014-2016) was made regarding the suspected cases of influenza in the general population and their agreement with suspected versus confirmed cases (patients) in pregnant patients. The incidence rate of being pregnant and sick with influenza was low in the three years: 2014 (0.23), 2015 (0.15) and 2016 (0.33) cases/person per year. However, the risk or probability of getting sick if you were pregnant versus not being pregnant was significant in the three years: for
2014 it was 1.6 times higher, for 2015 it was 2.7 times and for 2016 the probability of getting influenza was 1.9 times higher while being pregnant [10]. Pregnant women, like most people infected with influenza, feel sick for several days and then recover, but in them, it is known that influenza can lead to pneumonia or other complications, and even death [10-11].

In regard of getting a flu shot, is known for being the first and most important step to protect against it. Pregnant women should receive the injected vaccine and not the nasal spray vaccine. The literature highlights that the flu vaccine given during pregnancy helps protect the mother and her baby from this disease. And it has shown that vaccination reduces the risk of an acute respiratory infection in pregnant women in up to half of the cases. A study in 2018 reported that getting an influenza vaccination reduced a pregnant woman's risk of being hospitalized with a serious or complicated condition by an average of $40 \%$. Pregnant women who receive this vaccine are also helping to protect their babies from the disease during the first months after birth (cocoon effect), when they are too young to get vaccinated, because the mother transmits antibodies to the developing baby during her pregnancy, as such, vaccination protects the pregnant woman and her newborn (until they acquires an age to be vaccinated) from infections (level of evidence 1b). Similarly, early treatment with Oseltamivir is associated with a reduced risk of severe disease. And although is known that antiviral treatment that can equally reduce the risk of complications (level of evidence $2 \mathrm{~b}$ ), we should understand that both, maternal health and the evolution of her pregnancy product (baby) can be severely affected by an influenza infection virus (level of evidence 2b) [12-15].

As such, the objectives of the present study are: To know the epidemiology of pregnant women in different seasons of influenza in the State of Mexico, from the pandemic to the 2018-2019 season; analyze the group of pregnant women vaccinated vs unvaccinated pregnant women and who of them got sick of influenza and needed hospitalization, and her co-morbidities.

\section{Material and Methods}

A review was carried out of both the SINAVE-SISVEFLU platform, as well as the concentrates that are in the ISEM epidemiology department of each of the influenza periods or seasons, from the 2009-2010 pandemic event until the 2018-2019 season. All cases reported as positive for some type of virus (cases collected through the epidemiological surveillance of the USMI [Influenza Monitoring Health Units]), which were confirmed by the state laboratory, are protected in Excel from 2009 pandemic event till date. These data collected by epidemiological week are in the Department of Mycobacteriosis and Communicable Diseases of the Department of Epidemiology of the ISEM (Institute of Health of the State of Mexico). These reports or records were based on the platform designed by the DGE (General Direction of Epidemiology) as part of the pandemic influenza sentinel surveillance strategy in our country, where the influenza season normatively 
begins in the epidemiological week 40 and ends in the 20 th week of the following year [16].

\section{Statistical Analysis}

The analysis of each of the indicated influenza seasons (2009-2010 to 2018-2019) was performed, calculating percentages and prevalence's regarding the number of women captured with suspected influenza and who were pregnant. From the latter, it was analyzed how many were vaccinated and how many did not receive the influenza vaccine, and equally in both groups which was the type of influenza virus that was isolate and who they needed hospitalization. Firstly determining simple and cumulative frequencies per influenza season, at the same time, measures of central tendency and dispersion of the distribution of pregnant women per season were analyzed.

\section{Result}

A review of the last ten seasons of influenza (2009-2010 to 2018-2019) was made with regard to the suspected cases of influenza in the general population of the State of Mexico and its agreement with suspected versus confirmed (sick) cases in pregnant women Of these we found that there were a total of 18971 suspected cases, of which 9826 were women, resulting in only 10 years, $2.8 \%$ of pregnant women (276 pregnancies). The highest number of women with suspected influenza was detected in four seasons, highlighting 2015-2016 followed by 2009-2010, 20132014 and 2018-2019 with 1814, 1723, 1394, 1327 suspicious cases respectively, of these, the number of pregnant women showed a very low prevalence in all seasons, so the number of cases regarding suspicious women was not relevant. However, a higher percentage of pregnancies prevailed in 2013-2014, 2017 2018 and 2015-2016 seasons with 60-14 and 60 pregnancies; with a low prevalence in the 2009-2010 and 2011-2012 seasons, being this of 0.020 and 0.019 respectively. In fact, as such, this agrees even with the overall total of pregnant women vs. the total influenza suspects, whose prevalence which 0.028 .

In these 10 seasons of influenza in the State of Mexico we could see that although it is true that the number of pregnant women was not high except in 2013-2014 and 2015-2016 with 60 pregnancies respectively, contrasts with the number of pregnant women who received a vaccine against influenza, which was very low compared to the number of those not vaccinated, 40 and 46 respectively in those seasons. Regarding the vaccination of the 276 pregnant women, $186(67.3 \%)$ were not vaccinated vs of the vaccinated women who were only 82 (29.7\%). Vaccination status was unknown only in $2.8 \%$ ( 8 cases). Regarding the viruses identified in both vaccinated and unvaccinated pregnant women in each of the ten seasons, we observe that in the global total of pregnant women we found that $186(67.3 \%)$ were not vaccinated against influenza, of which 68 (36.5\% [68/186]) were positive for some type of virus. Predominating pandemic influenza AH1N1 (58.8\%), followed by influenza AH3 (25\%), Influenza B and finally influenza A with $10.2 \%$ and 5.8\% respectively. It should be noted that from the 2013-2014 season the lineage of influenza type B virus was identified, and those identified in the 10 seasons stood out the B-Victoria virus with 6 isolates and only 1 of B-Yamagata virus. As for the women who did receive a flu vaccine, when we analyzed their global, we found that only 82 of 276 were vaccinated, resulting in a percentage of $29.7 \%$, of which $81 / 82(98.7 \%)$ were positive for some type of virus. In this group, also predominated pandemic influenza AH1N1 with 42 isolates (51.8\%), followed by influenza AH3 (25.9\%), influenza B and influenza A with $17.2 \%$ and $4.9 \%$ respectively. And as for the B virus lineage, 6 of the Victoria type and 2 of the Yamagata type were identified. Regarding the percentage of the total virus identified (81) in pregnant women vaccinated (276), in all seasons was $29.3 \%$.

The distribution of both groups of pregnant women who those were not vaccinated vs. those that did get vaccinated and their relationship in terms of positivity of having acquired influenza and the type of virus identified and their relationship by the season analyzed are better. Regarding the impact of the disease that represented the risk of meriting the hospitalization of all those pregnant women with suspected influenza vaccinated or not, we observed that 49 of the 82 vaccinated were hospitalized $49(59.7 \%)$ while with the unvaccinated (186) was a higher rate of hospitalization (130 [69.8\%]), if we compare it with the positive hospitalized vaccinated we see that $60.4 \%$ merited real hospital admission, compared with the positive unvaccinated in which 191\% required hospital admission. It should be noted that in the global of the 276 pregnant women, most were hospitalized in the third trimester (27 of those vaccinated vs 49 unvaccinated); followed by the second trimester with 16 and 49 admissions of pregnant women vaccinated vs unvaccinated) and finally the first trimester was where they were least hospitalized (7 vs 32 between vaccinated and unvaccinated). As for the associated co morbidities for vaccinated pregnant women, we found 20 events, including 1 COPD, 1 systemic arterial hypertension, 2 HIV, 2 diabetes mellitus, 2 with heart disease, 3 asthmatics, 4 with positive smoking and 5 with obesity. It should be noted that one of them was diabetic and asthmatic, another with heart disease and positive smoking, one more with six co morbidities (Diabetic, with COPD, asthmatic, hypertensive, with HIV and heart disease). As for unvaccinated pregnant women, we found 45 co morbidities, these being 4 HIV, 2 diabetics, 3 with heart disease, 6 with positive smoking, 9 with high blood pressure, 10 with asthma and 11 obese. In this group, there were three patients with several co morbidities; 1 with hypertension, obesity and heart disease; another with diabetes mellitus, obesity and smoking, four with asthma, HIV and obesity, and two with asthma, hypertension, and obesity. These events and their relationship with the season analyzed are shown in Figure 1. In respect to the distribution for each season analyzed and the relationship of both groups of pregnant women (vaccinated vs. unvaccinated) who were hospitalized for suspected influenza and that was finally positive in terms of infection by some type of influenza virus identified, as well as the fact of only having received ambulatory management 
or even having died from influenza, we found that in the group of vaccinated of the 81 positive 32 (39.5\%) were managed on an outpatient basis with Oseltamivir. And on the unvaccinated group, although it is true that the majority were hospitalized, only 54/186 (29\%) patients received Oseltamivir on an outpatient basis. It is striking that in this group it was the only one where there were 2 deaths, which were recorded in the 20132014 season. These distribution events are shown in Figure 2.

Figure 1: Graphical representation by the season analyzed of vaccinated vs. unvaccinated pregnant women and their association with the hospitalization trimester and if they have o not a co morbidity. Note that in both groups the highest number of hospitalized cases was in the third trimester and co-morbidities were presented in greater numbers in the unvaccinated pregnant women. As well as the fact that of 82 vaccinated, 81 were positive for some type of influenza virus.

\section{Analysis}

According to the World Health Organization (WHO) it is known that pregnant women are in the high-risk group to present serious complications if they suffer an influenza event from any of the related viruses but especially if it is the type AH1N1 that usually gets complicated by bacterial pneumonia symptoms that threaten their life and could even die. This, as already mentioned favored by the physiological, immunological and mechanical changes that occur during the same pregnancy, and which is increased if the pregnant woman has any co morbidity $[2,17,18]$. It is striking that both the percentage and the prevalence of pregnant women in relation to the number of suspected cases in each season and even in the total of the 10 seasons were very low, This contrasts with the fact that the State of Mexico is considered as one of the states with the highest number of population and births, but we believe that this is due to low attendance at health centers or other levels of primary care of pregnant women, or that there is an under-registration in their recruitment, especially in communities where they are still assisted by midwives. In the global analysis when comparing vaccinated vs. unvaccinated we found that non-immunization predominated, since off from 276 pregnant women only $82(29.7 \%)$ received the vaccine vs $186(67.3 \%)$ who did not receive it. Although in 8 cases $(2.8 \%)$ their immunization status was unknown, this percentage is not significant. This association where the number of viruses that were most frequently identified by season is also analyzed. In this regard, we observe that, as reported in the literature, the AH1N1-PDM type predominated both in the unvaccinated pregnant woman (40/68 cases [58.8\%]) and in the vaccinated (42/81 cases [51.8\%]), representing 55\% of the total influenza virus isolates (82/149). In relation to the other three types of virus (AH3, $\mathrm{B}$, and $\mathrm{A}$ ), there was no significant difference in their identification in pregnant women who were not vaccinated than vaccinated, secondly identifying AH3 with 38 cases (17 in unvaccinated vs 21 in vaccinated), followed by type B with 21 cases ( 7 in unvaccinated and 14 in vaccinated) and finally type A with 8 cases ( 4 in unvaccinated and 4 in vaccinated). It highlights the fact that the highest number of viruses identified was in vaccinated pregnant women with a difference of 13 more cases in total. When we analyze the pregnant women who required hospitalization, as well as their co morbidities and the quarter in which they were hospitalized, we observe that although it is true the largest number corresponded to the unvaccinated (130/186 [69.8\%]) vs the vaccinated (49 / 82 [59.7\%]), it should be noted that practically $49(100 \%)$ hospitalized were positive for some type of influenza virus unlike those not vaccinated who only $52.3 \%$ were positive for some type of virus, which seems to indicate that the vaccine does not reduce the risk of hospitalization associated with influenza (which has not been reported in the literature). However, when analyzing the co morbidities, we found that in the group of vaccinated there were fewer related conditions than in the group of the unvaccinated (20 vs 46 events respectively); highlighting in the vaccinated, conditions such as: COPD, hypertension, diabetes mellitus, heart disease, asthmatic, smoking and obesity, in this group stands out the fact that there was a pregnant woman with more than six co morbidities and two with more than one. And in the unvaccinated group, we found 45 co morbidities, highlighting: HIV, diabetes mellitus, heart disease, positive smoking, high blood pressure, asthma, and obesity. Empathizing in this 
group, that nine patients with more than two co morbidities, being the 2015-2016 season where a greater number of co morbidities were detected ( 29 events in total). This suggests that in the unvaccinated hospitalization was more commonly associated with co morbidities than with the same influenza disease. Even the two deaths registered in the unvaccinated were associated with severe asthma. What is consistent with data from the literature is that the highest risk of hospitalization in pregnant women is during the third trimester of pregnancy, and in this analysis we observe that both vaccinated (27 cases) and unvaccinated (49 cases) were in the third trimester of pregnancy, although it is noteworthy that in the unvaccinated women was an equal number (49 cases) of hospitalized, which in some series has also been indicated that the second trimester also has a high risk of hospitalization for influenza. These data are shown in Figure 1. Finally, when analyzing hospitalizations vs. outpatient management, we see that of the 82 vaccinated 32 (39\%) could be managed on an outpatient basis, and of the 186 unvaccinated only 54 (29\%) received outpatient treatment. If we compare outpatient management with the fact of hospitalization, in both cases (vaccinated and non-vaccinated) hospital management was required, and since the majority of hospitalization was between the second and third trimesters, this agrees with what has been noted in other studies, that pregnant women do better if their treatment in into the hospital than outpatient, and in fact none of the 49 hospitalized women positive for influenza virus died in any of the seasons. These data are shown in Figure 2.

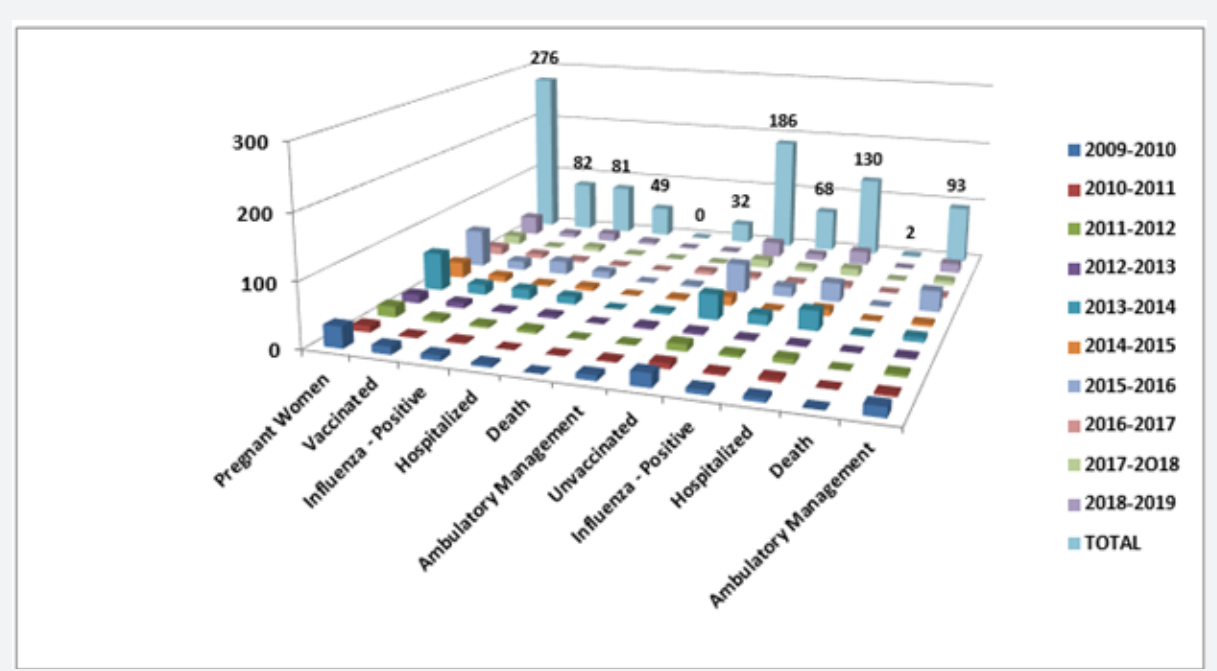

Figure 2: Graphical representation by season analyzed of pregnant women vaccinated vs non-vaccinated positive for influenza, hospitalized and their relationship with having died or having been managed on an outpatient basis. This graphic highlights the fact that of 276 pregnant women $125(45.2 \%)$ were managed on an outpatient basis. With only two deaths of the ten seasons analyzed of pregnant women.

\section{Conclusion}

According to the WHO, the pregnant woman is in the group of individuals at high risk to get sick and even to become seriously complicated during an infection-disease (level $2 \mathrm{~b}$ of evidence), apparently in this review we did not find a high risk of getting sick, however this may be biased because the capture of pregnant women does not represent the total of pregnancies in the state of Mexico. However, the data found indicate that the pattern of getting sick with influenza is the same as in the whole world; Associated with AH1N1 [12-13]. Likewise, finding that there was a greater number of fewer vaccinated (186/276 [67.3\%]) than those who were vaccinated $(82 / 276[29.7 \%])$, suggests that there is no advantage in receiving the influenza vaccine if will be are pregnant. Since in the global (of the 10 years analyzed), the associated risk of getting sick with influenza during pregnancy was $0.62(62.2 \%)$, however the relative risk of getting sick with influenza if it was vaccinated or not was 2.7 if was been vaccinated, with an OR of 140.6 times the probability of getting sick if she is vaccinated. Regardless, what impacts in the data obtained is that receiving an influenza vaccine does not reduce the risk of getting sick influenza, and nor does it reduce the risk of hospitalization in this group of pregnant women vaccinated. But since vaccination during pregnancy is safe, this activity should continue to be supported especially in those women who have some pre-existing co morbidity. And as such, since there is no study on the effectiveness of the influenza vaccine and associated hospitalizations for laboratory-confirmed influenza during pregnancy, more studies on that regard are necessary.

\section{Acknowledgment}

The authors thank all the doctors of the different municipalities of the State of Mexico for having opportunely captured their cases to the epidemiological surveillance platform for influenza. And to Andrea Coria Guerrero who was style corrector.

\section{Financing}

The expenses derivate from this publication were taken by each of the authors. 


\section{Conflicts of Interest}

The authors express that in the elaboration of this work weren't generated any conflicts of interest related to it.

\section{References}

1. Benedetti TJ, Valle R, Ledger WJ (1982) Antepartum pneumonia in pregnancy. Am J Obstet Gynecol 144(4): 413-417.

2. Rasmussen SA, Jamieson DJ, Uyeki TM (2012) Effects of influenza on pregnant women and infants. Am J Obstet Gynecol 207 (3 Suppl): S3-S8.

3. Jamieson DJ, Rasmussen SA (2019) Seasonal influenza and pregnancy.

4. Español (2019) Pregnant Women \& Influenza (Flu). Center for disease control and prevention, USA.

5. Jamieson DJ, Theiler RN, Rasmussen SA (2006) Emerging infections and pregnancy. Emerg Infect Dis 12(11): 1638-1643.

6. Cox S, Posner SF, McPheeters M, Jamieson DJ, Kourtis AP, et al. (2006) Hospitalizations with respiratory illness among pregnant women during influenza season. Obstet Gynecol 107(6): 1315-1322.

7. Rogers VL, Sheffield JS, Roberts SW, McIntire DD, Luby JP, et al. (2010) Presentation of seasonal influenza A in pregnancy: 2003-2004 influenza season. Obstet Gynecol 115(5): 924-929.

8. Centers for Disease Control and Prevention (CDC) (2009) Novel influenza A (H1N1) virus infections in three pregnant women - United States April-May 2009. MMWR Morb Mortal Wkly Rep 58(18): $497-$ 500

9. Calvo-Aguilar O, Canalizo-Mendoza YR, Hernández-Cuevas MJ (2011) Influenza AH1N1 en población obstétrica de un Hospital General de Oaxaca. Ginecol Obstet Mex 79(6): 344-350.

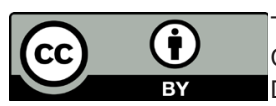

This work is licensed under Creative Commons Attribution 4.0 License DOI: 10.19080/JGWH.2019.17.555961
10. Coria LJ, Ortiz GE, Mirón CX, Pla EE (2018) Prevalencia e incidencia de influenza en la mujer embarazada durante las últimas tres temporadas de la misma (2013-2014; 2014-2015; 2015-2016). Arch Invest Matern Infant 9(2): 43-48.

11. Coria LJ, Ortiz GE, Mirón CX, Dávila ChE, Pla EE, et al. (2017) Morbilidad de la influenza en el Estado de México: a siete años de la pandemia. Neumol Cir Tórax 76(1): 17-23.

12. Thompson MG, Kwong JC, Regan AK, Katz MA, Drews SJ, et al. (2019) Influenza vaccine effectiveness in preventing influenza associated hospitalizations during pregnancy: a multicountry retrospective test negative design study, 2010-2016. Clin Infect Dis 68(9): 1444-1453.

13. Meijer WJ, vanNoortwijk AGA, Bruinse HW, Wensing AMJ (2015) Influenza virus infection in pregnancy: areview. Acta Obstet Gynecol Scand 94(8): 797-819.

14. Ortiz JR, Neuzil KM (2017) Influenza immunization of pregnant women in resource-constrained countries: an update for funding and implementation decisions. Curr Opin Infect Dis 30(5): 455-462.

15. Madhi SA, Cutland CL, Kuwanda L (2014) Maternal Flu Trial (Matflu) Team. Influenza vaccination of pregnant women and protection of their infants. N Engl J Med 371: 918-931.

16. Sinave (2012) Secretaría de Salud, Grupo Técnico Interinstitucional del Comité Nacional para la Vigilancia Epidemiológica (CONAVE). Lineamientos para la vigilancia epidemiológica de influenza, México, p. 4-35.

17. Rasmussen SA, Jamiesson DJ, Bresu JS (2008) Pandemic influenza and pregnant women. Emerg Infect Dis 14(1): 95-100.

18. Torres-Ramírez A (2010) La influenza pandémica A (H1N1) en mujeres embarazadas. Artículo de revisión, Ginecol Obstet Mex 78(2): 121-127.

\section{Your next submission with Juniper Publishers will reach you the below assets}

- Quality Editorial service

- Swift Peer Review

- Reprints availability

- E-prints Service

- Manuscript Podcast for convenient understanding

- Global attainment for your research

- Manuscript accessibility in different formats

( Pdf, E-pub, Full Tsext, Audio)

- Unceasing customer service

Track the below URL for one-step submission

https://juniperpublishers.com/online-submission.php 